\title{
A Study of Short-term Effect Measurement for Information Publication in Government Microblog
}

\author{
Ran Tang ${ }^{1}$, Zhenji Zhang ${ }^{1}$, Xiaolan Guan ${ }^{2}$ and Lida Wang ${ }^{3}$ \\ ${ }^{1}$ Beijing Jiaotong University, \\ ${ }^{2}$ Beijing Institute of Graphic Communication \\ ${ }^{3}$ China Software Testing Center
}

\{10113410,zhjzhang and0811310\}@bjtu.edu.cn andwanglida9@126.co

\begin{abstract}
From the microcosmic point of view, based on AISAS model, aiming at jear cased and data of government microblog information publication, this articte mokes a quantitative empirical research using correlation analysis and regression andysis and construct a quantitative model to measure the short-term effect of government microblog information publication. The measurement model lay a theoretical basis for improving effect of government microblog information.

Keywords: Government microblog, effect of information publication, AISAS model
\end{abstract}

\section{Introduction}

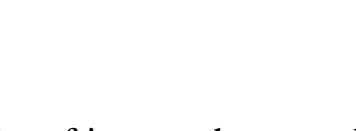

Development and popularity of internet have made the world become a public information platform. In 2011, as "the first year of government microblog" in China, government microblog passed into the explosive development stage, within a short time, government microblog has been an important platform and channel of governing online. Government microblog has lots of adyantages such as real-time, convenient and fast, open, close to the masses. Govemnent microbiog play a positive part on social management innovation, government information publicity, direction of public opinion, listening attentively to the voice of common people, building government image [1]. However, while the government is using microblog to make information publication, study of publication effect measurement is quite lacking

From the practice of government microblog: first, from August 2009 when Sina Microblog was launched, it is more than three years so far, development of government microblog is still in the short-term, temporarily, there is not enough long-term practice data to support. Therefore, the study object of this article is the short-term effect of information publication in government microblog. Second, the short-term indicators and data can be easily visualized, we can collect the short-term information publication data from the microblog platform easily. Based on the above reasons, this article attempts to construct the quantity model of short-term effect of government microblog information publication on the basis of real cases and data of government microblog information publication, and get a measurement method of short-term effect of government microblog information publication from the microscopic point. 


\section{Theoretical Basis}

\subsection{AISAS Model}

From traditional era to Internet era, internet and mobile application get the explosive popularity. Aiming at the changes of consumers' lifestyle in internet and wireless application times, Diantong company proposed a new consumer behavior analysis model-AISAS model [2]. AISAS model considers that in the Web 2.0 marketing mode in internet times, consumers would share and influence other consumers initiatively after they coming into contact with the product or the service information and buying it. In this process, consumers go through five steps: attention, interest, search, action, share.

In the five steps: on the one hand, in steps of attention and interest: any microblog user could follow the tracks of enterprise microblog initiatively and directly by searching the product or the service of the enterprise [3]. Therefore, the attention-interest step gathering fans of enterprise microblog could be simplified;

On the other hand, in steps of search, action and share: (1) mifcroblog has the instant search function and can form a real-time accurate information search; (2) because of the flow import and API interface application of microblog and e-busines website, consumers could produce purchasing action by means of microblog directly; (3) every account on microblog platform has the function of share, such as publish, comment and transmit consumers can share the product or the service information on microblog platform. Based on theses, enterprise must interact with fans proactively and initiatively forming the search-action-share step and better marketing effect[4]. In the enterprise microblog marketing practice, AISAS model could be subdivided into two steps, attention-interest step gathering fans and search-action-share step interacting with fans and between fans

However, there is no absolute bound between the two steps, gathering more fans in attention-interest step, it will be in more favor of enterprise microblog marketing in searchaction-share step; on the contrary, the beter in search-action-share step, more fans will be gathered in attention-interes slep[5]. Enterprise microblog marketing should be a circulation promotion process of attention-interest step and search-action-share step. Influence of enterprise microblog not paying attention to interacting with fans or interacting not well will show the tendency of smaller and smaller as AIDMA model describes and become lifeless. As a result, AISAS model can reflect the attenuation and amplification process of enterprise brand influence in microblog marketing from the positive and negative. Javaetal indicates that microblog users can easily form a close community with high correlation and interactivity, and form integral forware or reverse interactive impact on enterprise microblog marketing [6]. This reveals that in internet times enterprise turn the focus of the marketing activities from enterprise-centered to consumer-centered. Effect of enterprise marketing is not pure natural attenuation-trend along with the time, but existing natural attenuation trend and amplification trend because of consumers' interaction behavior like search, action and share [7]. Consumers' benign interaction can improve the actual conversion rate of the enterprise marketing activities.

Because of microblog's natural characteristics like information search, action, information share, AISAS model is very obvious in microblog. This article will construct the short-term effect measurement model of government microblog information publication on the basis of AISAS model. Aiming at multipolarity propagation path of microblog information, in the information spreading process microblog present the multipolarity of spreading step and mode: spreading in first stage present a mass-decentralized transmission state because of textual fragmentization and formal pertinence; spreading in second stage, because of deep linking and fusion with mass media, the spreading mode upgrades gradually into mass 
spreading state, and gets greater spreading effect [8]. The first stage could confirm attentioninterest step in AISAS model, and the second stage could confirm search-action-share step. However, because of complexity of government microblog users and microblog fans' multilevel amplification spreading, simple and visual AISAS model is lack of data interpretation. Therefore this article attempts to improve the model by means of data on the basis of the theory.

\subsection{Research Method}

Any microblog user's fan number, comment number, transmission number, microblog number and keywords of government microblog information publication can be inquired on microblog platform [9]. This provides great data support for empirical study of AISAS model and ensure the reality and reliability of case study. As a result, on the basis of AISAS qualitative theory and real case of Sina Microblog, this article will make a quantitative empirical research according to real case data and construct the short-term effect measurement model for information publication in government microblog. And also this article discusses the application range and many influencing factors of the measurement model.

\section{Model Construction}

\subsection{Research hypothesis}

In order to construct the relation model of government microblog information publication short-term effect and fan number, we use abscissa axis showing the time and use longitudinal symmetry vertical axis showing the nnfluence effect of government microblog information publication, as shown in Figure 1.

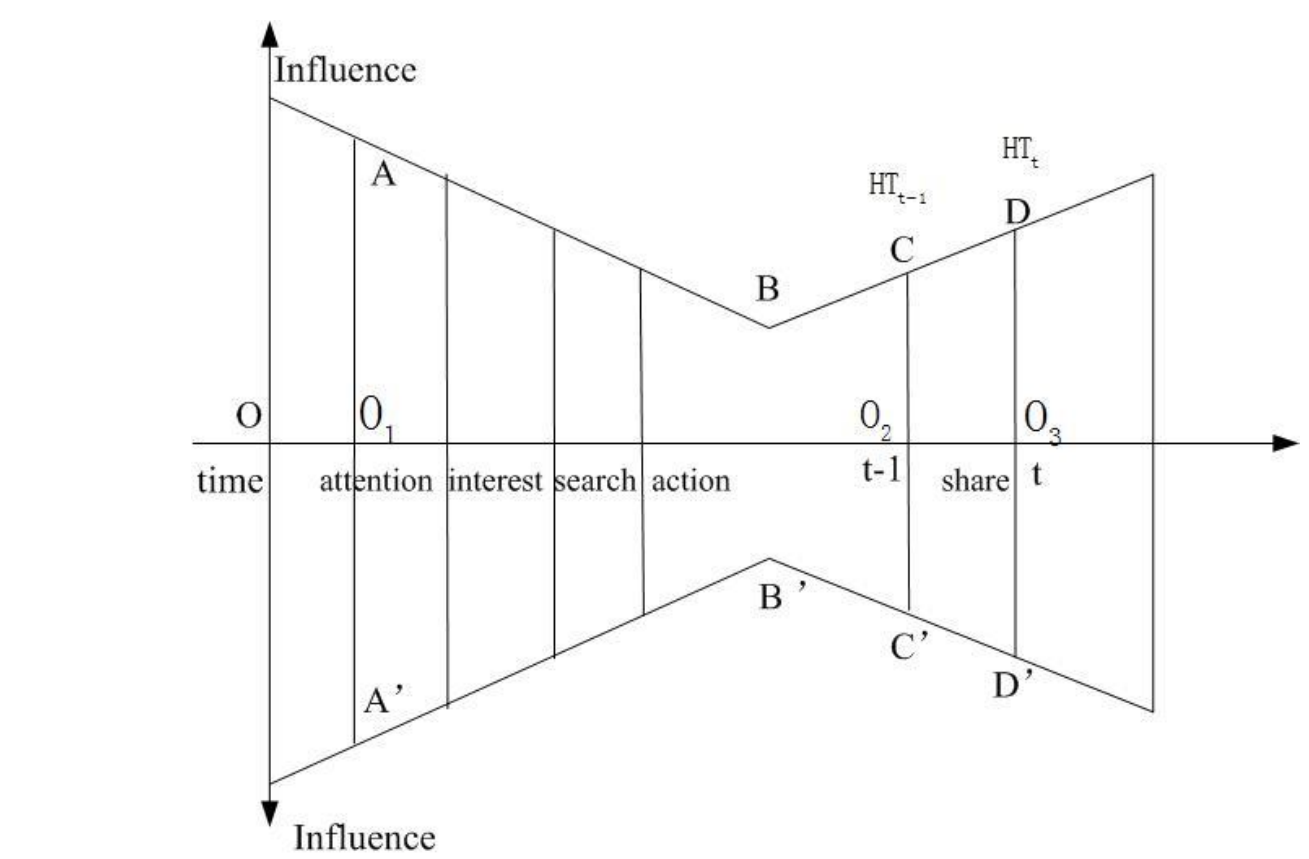

Figure 1. Local minimum and global optimization 
As in Figure 1, $\mathrm{O}$ indicates the time origin, $\mathrm{A}$ and $\mathrm{A}^{\prime}$ indicate the initial influence of information publication when government start to use microblog. Influence of government on microblog platform would present the tendency of weakening, in the natural attenuation process, government microblog go through attention-interest step and earlier stage of searchaction-share step. B and B' indicate that the influence of government microblog naturally attenuate to the lowest point. After this, short-term effect of government microblog information publication gradually emerge and form a positive cycle, the influence gradually rise and enter the action-share step. Take an observation time-point $t$ in the share step, when the influence of government microblog information publication is $\mathrm{D}$ and $\mathrm{D}$ '. $\mathrm{HT}_{i}$ indicates the accumulated influence between time-point $\mathrm{O}$ and $\mathrm{t}$; then take an observation time-point $\mathrm{t}$ 1 before $\mathrm{t}$, when the influence of government microblog information publication is $\mathrm{C}$ and $\mathrm{C}$ ', $\mathrm{HT}_{i-1}$ indicates the accumulated influence between time-point $\mathrm{O}$ and $\mathrm{t}-1 ; \mathrm{O}_{1}, \mathrm{O}_{2} \mathrm{O}_{3}$ are corresponding time-point of AA', CC', DD' on time axis. Variable definition ased in model constructing based on AISAS theory is shown in Table 1.

Table 1. AISAS model parameter of government microblog information publication

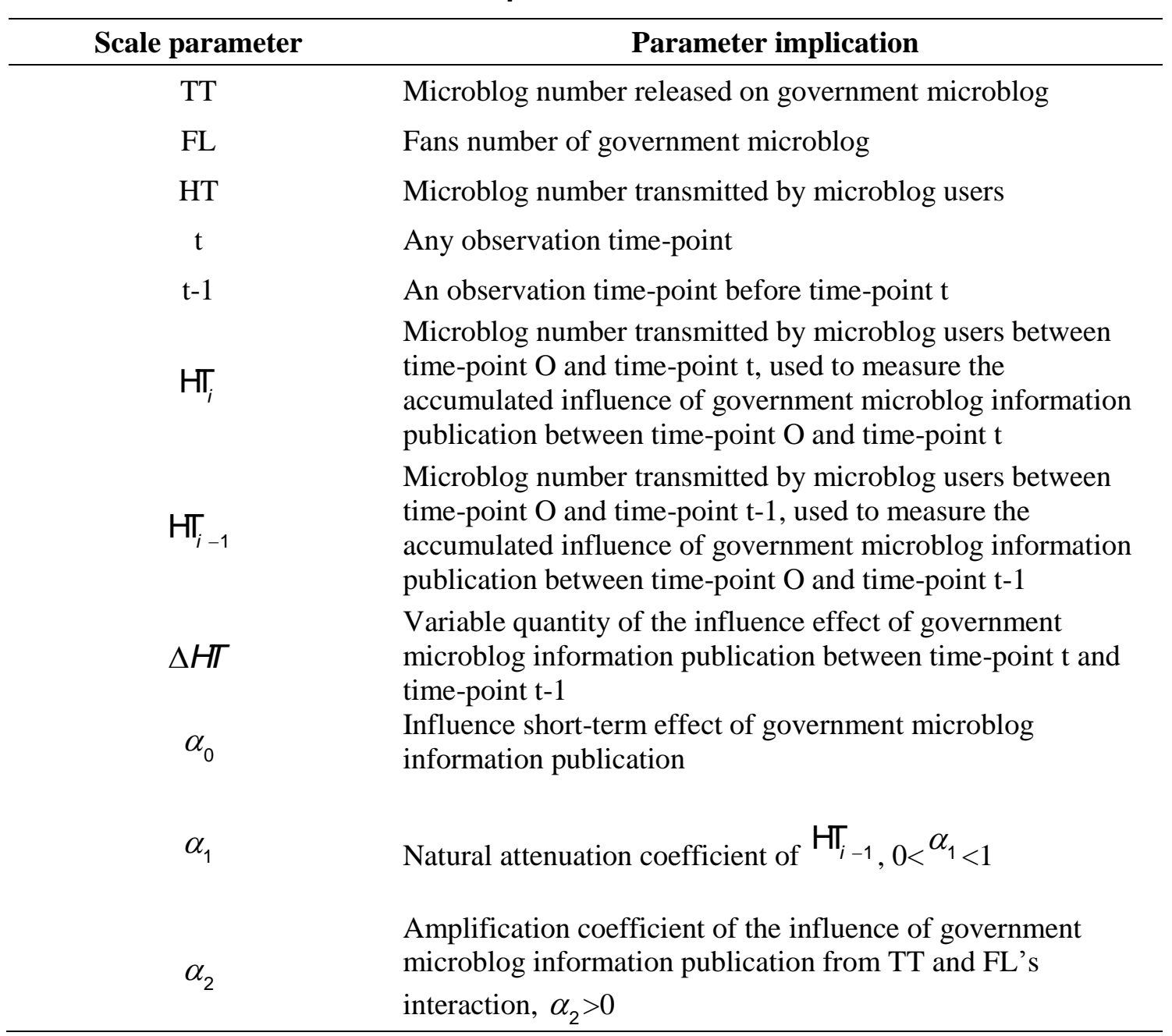


Operation mode of government microblog information publication is: government open the official microblog attracting early fans, and publish government information on microblog platform attracting fans to comment and transmit and attracting comments and transmission between fans. In this process, more and more fans pay attention to government microblog, at the same time, microblog participate in interaction with fans. As a result, a large number of government microblog are transmitted in multi-level and the effect of government information publication is improved. Data of government microblog number, government microblog fans can be inquired directly on microblog platform used to measure the influence effect of government microblog information publication.

By means of government microblog information publication, fans can search information they need by keywords and comment and transmit the information [10]. They share the experience on microblog platform immediately and produce abundance content. Also multilevel fans interact and form search-action-share step, attracting more fans to pay attention to government microblog. Attention- interest step and search-action-share step form a benign interaction so as to achieve the purpose of government information publication. So interactivity is a representative characteristic of government information publication in Web 2.0 times [11]. In the process of interaction between government and fans, on one hand, more active participation of government, more fans would pay attention to government microblog; on the other hand, more microblog information are publi hed, more fans would pay attention to government microblog, and interaction play more obvious function on short-term effect of government information publication. Therefore this article propose two related hypothesis:

H1: number of government microblog published and fans number are positive correlation.

$\mathrm{H} 2$ : the influence effect of government microblög information publication and number of government microblog and fans number are positive correlation.

In the whole AISAS stage, the influence has a natural attenuation effect. According to AIDMA theory, in the process that consumen getting product information to a final purchase, effect of enterprise marketing activities present a natural attenuation trend along with the time in the network transmission:[12]. In this article, we record all observation data once every day. Although the current data of information publication effect is smaller than the previous data, in the natural attenuation process, the greater the previous influence, the greater the current influence. Consequently this article propose another hypothesis:

H3: the current influence effect of information publication and the previous influence effect are positive correlgtion.

\subsection{Quantitative model}

Taking time-point $\mathrm{t}$ and $\mathrm{t}-1$, and accumulated influence effect of government microblog information publication between time-point $\mathrm{O}$ and $\mathrm{t}$ as $H T_{i}=S_{\mathrm{A}^{\prime} \mathrm{B}^{\prime} \mathrm{D}^{\prime} \mathrm{DBA}}$; accumulated influence effect of government microblog information publication between time-point $\mathrm{O}$ and t-1 as $H T_{i-1}=S_{A^{\prime} B^{\prime} C^{\prime} C B A}$, and during the observation period, variable quantity of the influence effect of government microblog information publication is $\Delta H T=H T_{i}-H T_{i-1}=S_{C^{\prime} D^{\prime} D}$. According to AISAS model, effect of government microblog information publication can be regard as the superposition of three parts: (1) attention-interest step, influence short-term effect of government microblog information publication is $\alpha_{0}$; (2) search-action-share step, by means of government microblog information publication, effect has been amplified, and formed by complex multi-level 
interaction between government microblog number TT and fans number FL, set as f(TT, FL); (3) natural attenuation effect of the influence in the whole AISAS step is attenuation effect of $\mathrm{HT}_{i-1}$ in observation period, set as $\alpha_{1} \cdot H T_{\mathrm{i}-1}, \alpha_{1}$ is natural attenuation coefficient, $0<\alpha_{1}<1$.

Seen by the multi-level communication complexity of government microblog information publication influence effect: (1) $\mathrm{HT}_{i-1}$ at any observation time-point has a hysteresis effect on $\mathrm{HT}_{i}, H T_{\mathrm{i}+1} \ldots \ldots$; (2) independence of TT and FL may lead to multicollinearity. So a first order autoregressive model of TT, FL, HT is constructed initially:

$$
H T_{i}=\alpha_{0}+\alpha_{1 \square} \cdot H T_{i-1}+f(T, F)
$$

\section{Empirical Study}

\subsection{Data sources}

This article is based on the short-term data of 6 typical government microblog on Sina Microblog in January 2013, and construct the short-term effect measurement model of government microblog information publication. Method of data collecting is as follows.

First of all, this article takes a high popularity government mictoblog - "Shanghai Fabu" as example to collect data. On November 28, 2011 "Shanghai Fabu" was put on line on Sina, and is one of the most concerned government mieroblog curently. Purpse of "Shanghai Fabu" is: gathering popularity by timely an authoritative information, clearing the way by the people's livelihood topic and highly interactivity, builing sizeable government microblog, forming the important platform for mass work in the new period initially, being a new channel to interact with mass for the government, pushing forward transparency, public and scientific, leading the current government mircoblog elegance.

This article mainly records microblog pumber, fans number and microblog transmission mumber of "Shanghai Fabu Crom January 1, 2013 to January 19, they are parameters TT, FL and HT. During Januar Ito January 19, 19 groups of valid data are collected. By regression analysis of the data this article construct the short-term effect measurement model of government microbrog information publication.

\subsection{Model parameters estimate}

Using software STATA 10.1 this article carries on the correlation detection of 19 groups of valid data of "Shanghar Fabu". Correlation coefficient of TT and FL are 0.992, H1 is verified; at the same time, in consideration of that FL has a straight amplification on HT, so this article replaces $\mathrm{f}(\mathrm{TT} / \mathrm{FL})$ with $\mathrm{f}(\mathrm{FL})$, in order to solve multicollinearity problem in formula (a).

According to H2: $\mathrm{f}(\mathrm{TT}, \mathrm{FL})=\mathrm{f}(\mathrm{FL})=\alpha_{2} \cdot \mathrm{F}$, substituted into the formula (a), we can get a firs order autoregressive model of short-term effect of government microblog information publication and fans number:

$$
H T_{i}=\alpha_{0}+\alpha_{1 \square} \cdot H \pi_{i-1}+\alpha_{2} \cdot F
$$

According to formula (b), using software STATA 10.1to fit a regression of 19 groups of valid data of "Shanghai Fabu", the results are as shown in Table 2. 
Table 2. Analytical statement of fitting regression of "Shanghai Fabu

\begin{tabular}{cccc}
\hline Observation data size : 19 & \multicolumn{3}{c}{ Squared value of R : 0.989 } \\
\hline & Coefficient & Statistic of t & Probability \\
$\mathrm{FL}$ & 0.015 & 2.689 & 0.011 \\
$\mathrm{HT}_{i-1}$ & 0.832 & 15.769 & 0.000 \\
$\alpha_{0}$ & 13.167 & 2.101 & 0.042 \\
\hline
\end{tabular}

(1) $R^{2}=0.989$, that means a high fitting degree of $H T_{i}=\alpha_{0}+\alpha_{1 \square} \cdot H T_{i-1}+\alpha_{2} \cdot F_{\text {; }}$;

(2) $\alpha_{0}=13.167, \alpha_{1}=0.832, \alpha_{2}=0.015$, and correspondent statistic of t are more than 2 , that means three variables are significantly different from zero, H2, H3 are verified from the data perspective;

(3) $H T_{i}=\alpha_{0}+\alpha_{1 \square} \cdot H T_{i-1}+\alpha_{2} \cdot F$, that means the first order autoregressive model of short-term effect of government microblog information publication and fans number can be accepted. The model can be described specifically as:

$$
H T_{i}=13.167+0.832 \cdot H T_{i-1}+0.015 \cdot F
$$

\subsection{Regression result analysis}

In 2010, Sina Microblog became the first choice of government microblog[17]. By contrastive analysis of different government microblog's regression test result, and discussion of important parameters, we can discuss the short-term effect measurement model more deeply. According to themodel $H T_{i}=\alpha_{0}+\alpha_{1} \cdot H T_{i-1}+\alpha_{2} \cdot F$, using software STATA 10.1, this article fit a regression of valid data of other 5 government microblog and test it, the results are as shown in Tabie 3.

Table 3. Analytical statement of fitting regression of 6 government microblog

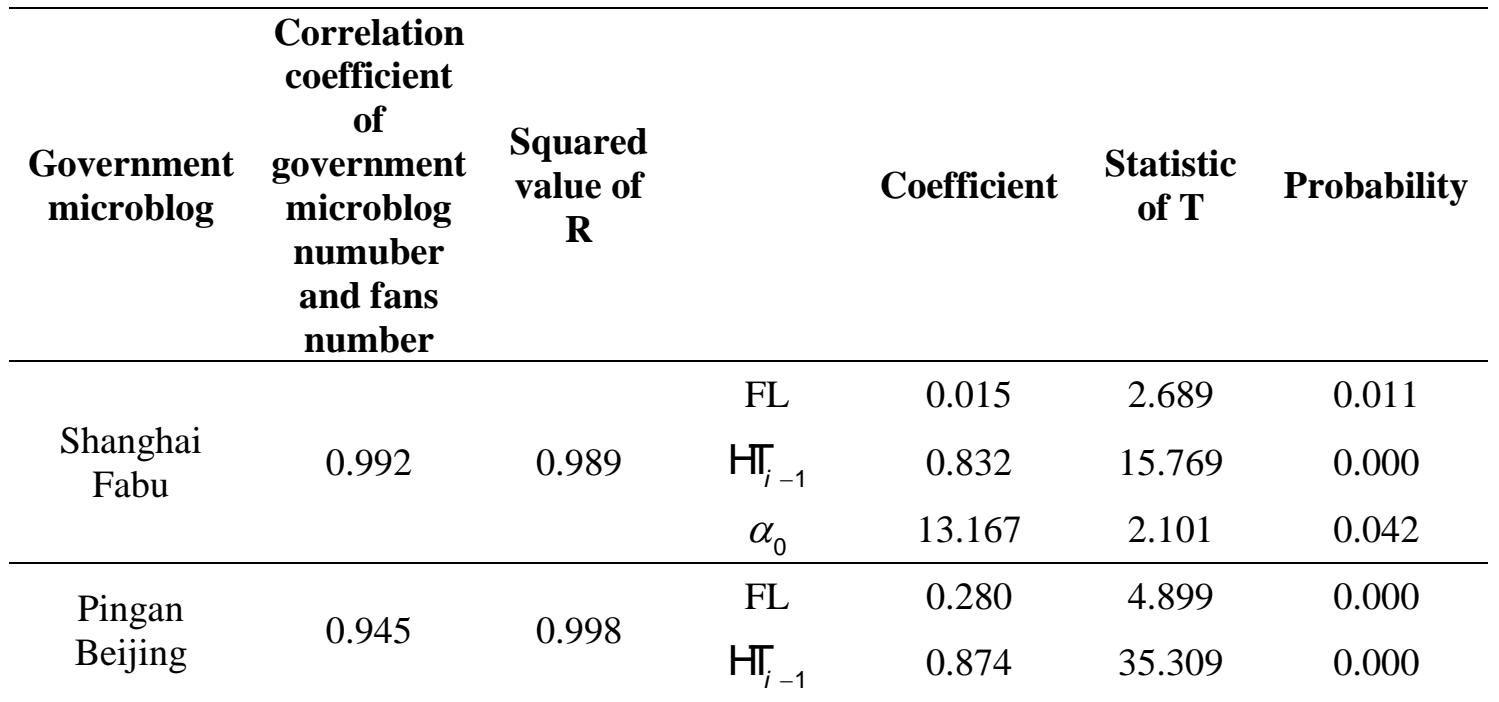




\begin{tabular}{|c|c|c|c|c|c|c|}
\hline $\begin{array}{c}\text { Government } \\
\text { microblog }\end{array}$ & $\begin{array}{c}\text { Correlation } \\
\text { coefficient } \\
\text { of } \\
\text { government } \\
\text { microblog } \\
\text { numuber } \\
\text { and fans } \\
\text { number } \\
\end{array}$ & $\begin{array}{c}\text { Squared } \\
\text { value of } \\
\mathbf{R}\end{array}$ & & Coefficient & $\begin{array}{c}\text { Statistic } \\
\text { of } \mathbf{T}\end{array}$ & Probability \\
\hline & & & $\alpha_{0}$ & -813.002 & -3.619 & 0.001 \\
\hline \multirow{3}{*}{$\begin{array}{c}\text { Nanjing } \\
\text { Fabu }\end{array}$} & \multirow{3}{*}{0.971} & \multirow{3}{*}{0.999} & FL & 0.210 & 1.531 & 0.134 \\
\hline & & & $\mathrm{HT}_{i-1}$ & 0.916 & 19.761 & 0.000 \\
\hline & & & $\alpha_{0}$ & -2279.52 & -1.431 & 0.159 \\
\hline \multirow{3}{*}{$\begin{array}{l}\text { Humen } \\
\text { Lvyou }\end{array}$} & \multirow{3}{*}{0.995} & \multirow{3}{*}{0.998} & $\mathrm{FL}$ & & & 0.016 \\
\hline & & & $\mathrm{HT}_{i-1}$ & & & 0.000 \\
\hline & & & $\alpha_{0}$ & & & 0.433 \\
\hline \multirow{3}{*}{ Ningbo Fabu } & \multirow{3}{*}{0.949} & \multirow{3}{*}{0.993} & & & .249 & 0.000 \\
\hline & & & & & -1.119 & 0.278 \\
\hline & & & & & -5.129 & 0.000 \\
\hline \multirow{3}{*}{$\begin{array}{l}\text { Weibo } \\
\text { Yunnan }\end{array}$} & \multirow{3}{*}{0.969} & & & & 1.529 & 0.132 \\
\hline & & & & 0.914 & 19.759 & 0.000 \\
\hline & & & & -248.32 & -1.429 & 0.157 \\
\hline
\end{tabular}

The results of the empirical study of the measurement model are as follows:

(1) $\alpha_{2}$ can be used to measure the short-term effect of government microblog information publication.

On government microblog "Pingan Beijing" during January 1, 2013 to January 19, $\alpha_{2}=$ 0.280 , far more than $\alpha_{2}=0.015$ of "Shanghai Fabu". This is in line with the actual situation information publication effect of "Pingan Beijing" is better and get the public's consistent high praise. In especial the special column "micro tip", provide the residents in Beijing t mely and accurate traffic message, greatly facilitate the residents' travel arrangements. Under normal circumstances, $\alpha_{2}$ is the most important index to measure the short-term effect of government microblog information publication.

(2) The greater $\alpha_{2}$ is, at the same time the smaller $\alpha_{0}$ is and even negative, the short-term effect of government microblog information is better.

When $\mathrm{t}=1, H \pi_{1}=\alpha_{0}+\alpha_{2} \cdot F$. Generally speaking, the greater $\alpha_{2}$ is, that means vitality of fans is higher, and it will make FL greater, $\alpha_{2} \cdot F$ greater. Therefore, in the 
first order autoregression model, $\alpha_{0}$ become smaller and even negative. $\alpha_{0}$ of "Pingan Beijing" is -813.002 , much smaller than $\alpha_{0}$ of "Shanghai Fabu" which is13.167. Under normal circumstances, combination of $\alpha_{2}$ and $\alpha_{0}$ can be used to measure the short-term effect of government microblog information publication preferably.

(3) $\alpha_{1}<0$ means government microblog information publication is distortion.

$\alpha_{1}$ is attenuation coefficient of $\mathrm{HT}_{i-1}, 0<\alpha_{1}<1$; but for "Ningbo Fabu", $\alpha_{1}<\mathrm{o}$, and $\alpha_{2}=0.857$, much greater than $\alpha_{2}=0.280$ of "Pingan Beijing", this means vitality of fans of "Ningbo Fabu" is higher than fans of "Pingan Beijing", this is not in line with the actual situation. The only explanation is that there are many completely inactive rans of "Ningbo Fabu", result in the distortion of government microblog information publication, $\alpha_{1}$ is negative.

\section{Conclusion}

From the microcosmic point, based on AISAS model, this article collect the real data of 6 government microblog on Sina and make a quantitatıve empirical research using correlation analysis and regression analysis. Aiming at the short-term effect of government microblog information publication this article construct the quantitative model-a first order autoregression model of the short-term effect of goyernment microblog information publication and fans number, and test the rationality of the model. Quantitative model in this study provides a measurement scheme to measure the short-term effect of government microblog information publication.

The results prove that: in the peocess of government microblog information publication, the current influence effect of information publication and the previous influence effect are positive correlation; the influence effect of government microblog information publication and number of government microblog and fans number are positive correlation. At the same time, coefficients in the measuremen model have a corresponding meaning.

For amplification coefficient $\alpha_{2}\left(\alpha_{2}>0\right)$ : (1) $\alpha_{2}$ can be used to measure the short-term effect of government microblog information publication; (2) the greater $\alpha_{2}$ is and the smaller $\alpha_{0}$ is and ever negative, short-term effect of government microblog information publication is bêtten For attenuation coefficient $\alpha_{1}\left(0<\alpha_{1}<1\right): \alpha_{1}<0$ means government microblog information publication is distortion. Amplification coefficient in the model can be used to measure the short-term effect of government microblog information publication, attenuation-coefficient can be used to measure true and false of government microblog infonmation publication.

\section{Acknowledgements}

This paper is supported by the subject of "Beijing Jiaotong University Doctor Innovation Funds" (NO. 2012YJS052). 


\section{References}

[1] M. Mushfiqur, R. Chowdhury, S. Alam, Z. Iqbal and J. Noll, "Sociality brings Security in Content Sharing", Journal of Computers, vol. 5, no 12, (2010), pp. 1839-1846.

[2] L.Y. Zhang, "Diantong reconstruct consumer's behavior model”, Journal of Modern Advertisement, vol. 2, (2007).

[3] K. Fumito, "The modeling of AISAS marketing process", Journal of System Dynamics, vol. 8, (2009), pp. 95-102.

[4] S. Huang, "First exploration of microblog multipolariy spreading mode", Journal of Young Reporters, vol. 374, no. 4, (2010).

[5] E. Mischaud, "Twitter: Expression of the whole self -- An investigation into user appropriation of a webbased communications platform", London School of economics and political science, the University of London, (2007).

[6] K. Faizullah, K. Surat and B. Zhang, "Public-private Partnerships and E-government", Journal of Advances in Intelligent and Soft Computing, vol. 115, no. 2, (2012).

[7] K. H. Golam and M. A. Akhtar, "Evaluation of Customer Oriented Success Factors in Mobile Commerce Using Fuzzy AHP”, Journal of Industrial Engineering and Management, vol. 4, no 2, (2011).

[8] Z. X. Lin and H. Chen, "The applications of creative SMS value-added service based on web2.0 concept", Journal of Science and Engineering Technology, vol. 6, no. 3, (2010), pp. 55-64.

[9] Z. Chenyi, S. Jianling and D. Yiqun, "Topic Mining for Microblog Based on MB-LDA Model", Journal of Computer Research and Development, vol. 48, no. 10, (2012), pp. 1795-1802.

[10] B. J. Jansen, M. Zhang, K. Sobel and A. Chowdury, "Twitter power: tweets as electronic word of mouth", Journal of The American Society For Information Science And Technology, vol.60, no. 11, (2009), pp. 21692188.

[11] L. Jie, Z. Xin and C. Wei, "SINA Microblog Data Retrieval”, Journa Cof Tsinghua University (Sci \& Tech), vol. 51, no. 10, (2011), pp. 1301-1305.

[12] C. Liwen and C. Tung-Liang, "Use of Twitter for Formative Eyaluation: Reflections on Trainer and Trainees Experiences", British Journal of Educational Technology, yo1 43, no. 2, (2012), pp.49-52.

[13] K. Nalini, V. D. Geest and M. Thea, The Tension Between User-centered Design and E-government Services", Journal of Behaviour and In ormationTechnology, vol 31, no 3, (2012).

[14] C. S. Ong and S. W. Wang, "E-Service and Organizational Change: A Process Model”, Journal of Electronic Commerce in Organizations, vol. 9, no 3, (2011).

[15] T. Zhanwei and Z. Qinpu, "Empirical Analysis Of Mircroblog Information Flow Features Based on Complex Network Theory", Journal of Advances in Information Sciences and Service Sciences, vol. 4, no. 7, (2012), pp. 163-171.

[16] M. Xin, H. Wu and Z. Niu, “A Quick Emergency Response Model for Micro-blog Public Opinion Crisis Based on Text SentimentIntensity" Journal of Software, vol. 7, no. 6, (2012), pp. 1413-1420.

[17] Z. Li, Y. Ruixi and G. Xiaohong, "Bursty Propagation Model for Incidental Events in Blog Networks", Journal of Software, vol. 20, no5, (2009), pp. 1384- 1392.

[18] V. Weerakkody S. Jones and E.Olsen, "E-government: a comparison of strategies in local authorities in the UK and Norway", Internationat Journal of Electronic Business, vol. 5, no. 2, (2007).

[19] H. Xu, H. H. Teo, B. C.Y. Tan and R. Agarwal, "Effects of Individual Self-Protection, Industry SelfRegulation, and Government Regulation on Privacy Concerns: A Study of Location-Based Services”, Journal of Information Systems Research, vol. 23, no 2. (2012).

[20] N. Zhang, Guo, X.G. Chen and P.Y. K. Chau, "Impact of Perceived Fit on E-government User Evaluation: A Study with A Chinese Cultural Context”, Journal of Global Information Management, vol. 17, no. 1, (2009).

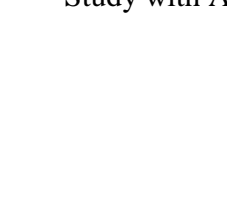

